\title{
INDICES OF OXIDATIVE STRESS AND LIVER INJURY IN ALCOHOLIC
} LIVER DISEASE

Pinky Garg ${ }^{* 1}$, Saroj Choud hary ${ }^{2}$, Montosh Chakraborty ${ }^{3}$, Karthikeyan P 4.

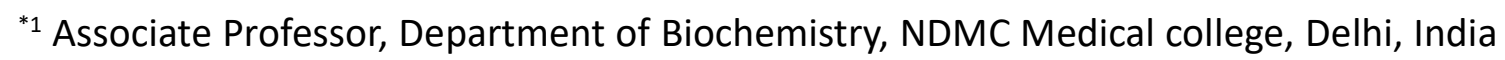

${ }^{2}$ Assistant Professor, Department of Biochemistry, NDMC Medical college, Delhi, India

${ }^{3}$ Assistant Professor, Department of Biochemistry, Andaman and Nicobar Islands Institute of Medical Sciences, Port Blair, India.

${ }^{4}$ Senior Resident, Department of Biochemistry, All India Institute of Medical Sciences, New Delhi, India.

\section{ABSTRACT}

Background: Alcohol is the most common substance of abuse causing alcoholic liver disease (ALD) becoming the major public health problem worldwide. During metabolism of alcohol in the liver reactive oxygen species (ROS) are generated which cause lipid peroxidation leading to hepatotoxicity. The body's natural antioxidant defense system against free radicals is depleted by alcohol consumption.

Purpose of the study: This study was designed to know how alcohol metabolism increases oxidative stress and inflicts damage to liver and decreases the efficiency of antioxidant defense system in patients of ALD.

Materials and Methods: It was a case control study of 50 patients clinically and radiologically diagnosed of ALD compared with 50 age and sex matched normal healthy controls. All the subjects were interviewed as per the proforma designed for alcoholics. This study compared lipid peroxidation product in the form of Malondialdehyde (MDA) levels and antioxidant status in the form of reduced Glutathione (GSH) levels in ALD patients with healthy controls.

Results: The results were analyzed statistically and showed that the level of MDA of cases was significantly higher ( $p$ value<0.0001) than the control group. In comparison to normal healthy persons the GSH levels of patients of ALD showed significant decrease ( $p$ value<0.0001). Conclusion: Our results reflected increased oxidative stress in ALD and based on these findings we can explain beneficial role of antioxidant supplementation along with alcohol abstinence in delaying the progression of ALD.

KEY WORDS: Alcoholic liver disease, Reactive oxygen species, Malondialdehyde, Glutathione.

Address for correspondence: Dr. Pinky Garg, Associate Professor, Department of Biochemistry, NDMC Medical College, Delhi, India. E-Mail: dr.pinky18@gmail.com

\begin{tabular}{|l|lc|}
\hline \multicolumn{3}{|c|}{ Online Access and Article Informtaion } \\
\hline Quick Response code & \multicolumn{1}{|c|}{ International Journal of Integrative Medical Sciences } \\
\cline { 2 - 3 } & \multicolumn{2}{c|}{ www.imedsciences.com } \\
\cline { 2 - 3 } & Received: 17-07-2017 & Accepted: 20-08-2017 \\
Dol: 10.16965/jims.2017.107 & Reviewed: 17-07-2017 & Published: 05-09-2017 \\
\hline Source of Funding: Self & \multicolumn{2}{c|}{ Conflicts of interest: None } \\
\hline
\end{tabular}

\section{BACKGROUND}

Liver disease related to alcohol consumption fits into one of the three categories: fatty liver, alcoholic hepatitis, or cirrhosis. Liver is the major alcohol processing organ. Chronic heavy drinking induces liver injury and results in ALD, even irreversible alcoholic liver cirrhosis [1]. It remains one of the most common causes of chronic liver disease [2]. Approximately 10-35\% heavy drinkers develop alcoholic hepatitis and $10-20 \%$ develop cirrhosis [3].

Most of the alcohol, a person drinks is eventually broken down by liver and products generated are more toxic than alcohol itself. A group of metabolic 
products called free radicals can damage liver cells and promote inflammation. The body's natural defense against free radicals (e.g. antioxidants) can be inhibited by alcohol consumption leading to liver damage [4]. It also activates hepatic macrophages that produce cytokines which induces mitochondria to increase production of ROS. This oxidative stress promotes liver cell necrosis and apoptosis which are exaggerated in the alcoholic who is deficient in antioxidants such as glutathione and vit $E$ [5-6]. The primary factors involved in the development of ALD are acetaldehyde, oxidative stress, hypoxia, membrane changes and the immune response [7]. Hepatocytes are the site of ethanol oxidation and ethanol induced injury.

Free radicals have a very short half-life, which makes them very hard to measure in the laboratory. Radicals can be measured using electron spin resonance and spin trapping methods. A commonly used alternate approach measures markers of free radicals rather than the actual radical. When a fatty acid is peroxidized it is broken down into aldehydes, which are excreted. Aldehydes such as thiobarbituric acid reacting substances (TBARS) have been widely accepted as a general marker of free radical production [8]. The most commonly measured TBARS is malondialdehyde (MDA) [9].This compound is a reactive aldehyde and is one of the many reactive electrophile species that cause toxic stress in cells and form covalent protein adducts which are referred to as advance lipoxidation end products (ALE), in analogy to advanced glycation end-products (AGE) [10]. The production of this aldehyde is used as a biomarker to measure the level of oxidative stress in an organism [11,12].

Antioxidants are formed within the body and can also be extracted from the food humans eat such as fruits, vegetables, seeds, nuts, meats, and oil. There are two lines of antioxidant defense within the cell. The first line, found in the fat-soluble cellular membrane consists of vitamin $E$, beta-carotene, and coenzyme $Q$ [13]. Of these, vitamin $E$ is considered the most potent chain breaking antioxidant within the membrane of the cell. Inside the cell water soluble antioxidant scavengers are present.
These include vitamin C, glutathione peroxidase, superoxide dismutase (SD), and catalase [14].

Glutathione is produced in the human liver and plays a key role in intermediary metabolism, immune response and health, though many of its mechanisms and much of its behavior await further medical understanding. Glutathione is found in two forms, a monomer that is a single molecule of the protein, and a dimmer that is two of the single molecules joined together. The monomer is called reduced glutathione, while the dimer is also called oxidized glutathione. The monomer is the active form of glutathione. Oxidized glutathione is broken down to the single molecule by an enzyme called glutathione reductase [15].

The present study was planned to see oxidative stress in patients of ALD in socioeconomic belt of Amritsar, Punjab. Antioxidants are likely to provide beneficial effects on hepatocytes via desensitization against oxidant stress while inhibiting primary mechanism for expression of pro-inflammatory and cytotoxic mediators.

\section{MATERIALS AND METHODS}

Study design: It was a case control prospective study. The patients and the controls were screened for Malondialdehyde (MDA), reduced Glutathione (GSH), Aminotransferases (AST, ALT) and gammaglutamyl transpeptidase (GGT) levels.

Study group: The patients included in the study were nonsmokers and consuming alcohol for 10 20 years \& suffering from Alcoholic liver disease attending the O.P.D and the wards of Medicine Department. 50 male patients in the age group of 40-70 years diagnosed on the basis of history, clinical symptoms and imaging studies were selected for the study. 50 normal healthy persons, age and sex matched not consuming alcohol from the same population served as control. The patients suffering from diabetes mellitus, malignancies, tuberculosis, hypertension, hepatitis, coronary artery disease and the patients taking antioxidant drugs were excluded from the study. Informed consent was procured from all of them for participation in the study and the study was approved by the ethics committee of the Medical College. 
Biochemical analysis: Following investigations were carried out by given methods.

1. Serum MDA level estimation: By method of Beuge and Aust (1978) [16]

2. Blood glutathione level estimation: By Beutler et al method using 5-5'dithione 2-nitrobenzoic acid (DTNB) [17]

3. Serum AST,ALT \&GGT level estimation: By kinetic IFCC method [18] by using kits

Statistical analysis: The results were expressed as mean $\pm S$.D of each variable. The comparison was done by using student ' $t$ ' test on the number of variables for each parameter. The value of significance was evaluated with ' $p$ ' value. $P$ value less than 0.05 was considered as the significant.

\section{RESULTS}

The baseline characteristics of the patients (all males) are presented in Table 1. It was seen that mean age in both cases and controls was nearly same and no significant difference in age was found. The liver function tests were normal in all the 50 non-alcoholics. Comparison of plasma MDA and GSH levels among alcoholics and nonalcoholics is shown in table 2. The statistical analysis revealed that the mean MDA levels were significantly higher among alcoholics in comparison with non-alcoholics $(p<0.001)$ (Table 2 ). The mean GSH levels were significantly higher in non-alcoholics compared to alcoholics $(p<0.001)$.

In this study, the cases and controls were divided into 3 groups on the basis of age i.e. Group I, II, III. ALD was most predominant in the age group of $40-50$ yrs. There were 28 patients in the Group I. When the levels of liver function tests, MDA and GSH in patients were compared in different age groups, the difference was statistically not significant (Table 3).

Figure 1 shows distribution of the cases into 2 groups on the basis of duration of alcohol consumption i.e. Group I, II. Group I included 24 cases having duration of alcohol consumption d" 20 years. Group II included 26 cases having duration of alcohol consumption $>20$ years.

Fig 2 compares relation of various markers discussed above with the duration of alcohol consumption in cases and it was observed that there was no significant variation in various parameters under study with duration of alcohol consumption ( $p=$ Not Significant). It may be due to different amount of alcohol intake, nutritional and comorbidity factors.

Table 1: Baseline characteristics of study population and control subjects.

\begin{tabular}{|c|c|c|c|}
\hline variable & Alcoholic (Cases) & $\begin{array}{l}\text { Nonalcoholic } \\
\text { (Controls) }\end{array}$ & $P$ value \\
\hline Age (years) & $51.38 \pm 5.78$ & $52.02 \pm 7.94$ & $\begin{array}{c}p>0.05 \\
\text { not Significant }\end{array}$ \\
\hline AST(IU/L) & $88.90 \pm 42.78$ & $21.58 \pm 6.26$ & \multirow{4}{*}{$\begin{array}{c}\quad p<0.0001 \\
\text { Highly Significant }\end{array}$} \\
\hline ALT(IU/L) & $29.82 \pm 12.81$ & $17.94 \pm 9.81$ & \\
\hline GGT(IU/L) & $129.04 \pm 49.81$ & $16.38 \pm 12.20$ & \\
\hline AST/ALT ratio & $3.13 \pm 1.13$ & $1.49 \pm 0.78$ & \\
\hline
\end{tabular}

Table 2: Indices of oxidative stress in the study population.

\begin{tabular}{|c|c|c|c|}
\hline Variable & Cases & Controls & P value \\
\hline $\begin{array}{c}\text { MDA } \\
\text { (mMoles/L) }\end{array}$ & $5.70 \pm 1.42$ & $1.80 \pm 0.62$ & $\begin{array}{c}\text { p }<0.0001 \\
\begin{array}{c}\text { GSH } \\
\text { (mMoles/L) }\end{array}\end{array}$ \\
\hline
\end{tabular}

Table 3: Comparison of Mean Serum MDA, GSH, AST, ALT, GGT Levels in different age groups in Cases.

\begin{tabular}{|c|c|c|c|c|c|c|}
\hline $\begin{array}{c}\text { Age group } \\
\text { (in years) }\end{array}$ & $\begin{array}{c}\text { No of } \\
\text { Subjects } \\
\text { (Cases) }\end{array}$ & $\begin{array}{c}\text { Mean MDA } \\
\text { levels } \\
\text { (mMo/L) }\end{array}$ & $\begin{array}{c}\text { Mean GSH } \\
\text { levels } \\
\text { (mMol/L) }\end{array}$ & $\begin{array}{c}\text { Mean AST } \\
\text { levels (IU/L) }\end{array}$ & $\begin{array}{c}\text { Mean ALT } \\
\text { levels (IU/L) }\end{array}$ & $\begin{array}{c}\text { Mean GGT } \\
\text { levels (IU/L) }\end{array}$ \\
\hline $\begin{array}{c}\text { Groupl } \\
\text { (40-50) }\end{array}$ & 28 & $5.87 \pm 1.50$ & $0.54 \pm 0.24$ & $96.82 \pm 44.23$ & $32.21 \pm 13.94$ & $127.39 \pm 53.29$ \\
\hline $\begin{array}{c}\text { GrouplI } \\
\text { (51-60) }\end{array}$ & 14 & $5.38 \pm 1.44$ & $0.45 \pm 0.21$ & $84.29 \pm 39.48$ & $28.14 \pm 10.93$ & $124.21 \pm 41.76$ \\
\hline $\begin{array}{c}\text { Group III } \\
\text { (61-70) }\end{array}$ & 8 & $5.70 \pm 1.15$ & $0.47 \pm 0.23$ & $69.25 \pm 40.43$ & $24.38 \pm 10.72$ & $143.25 \pm 53.73$ \\
\hline
\end{tabular}

Fig. 1: Distribution of case according to the duration of alcohol consumption.
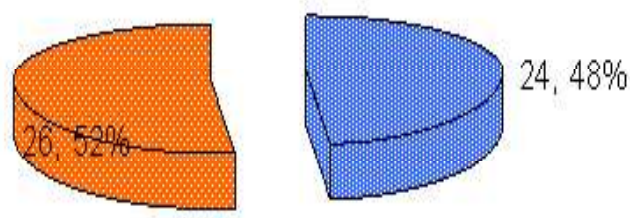

\section{DDuration $\leq 20 y r s \quad$ DDuration $>20$ yrs}

Fig. 2: Comparison of Mean Levels of various markers according to duration of alcohol consumption.

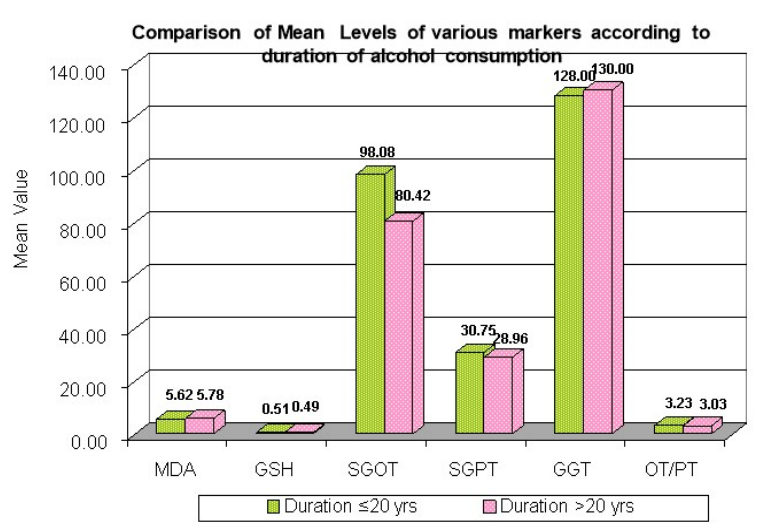




\section{DISCUSSION}

Alcoholic beverages and the problems they engender have been familiar in human societies since the beginning of recorded history. Liver is of vital importance in intermediary metabolism and in the detoxification and elimination of toxic substances. Since ALD has no specific clinical features and no specific laboratory tests are available for it, its diagnosis is currently based on drinking history, related laboratory assessments and imaging. Certainly, liver biopsy is the gold standard for diagnosis of ALD [19], but it is hard to use this invasive examination in this study. The Main aim of this study was to evaluate MDA and GSH in patients of ALD and to assess the utility of these parameters as markers of oxidative stress.

In this study, the cases and controls were divided into 3 groups on the basis of age. ALD was most predominant in the age group of 40 50 yrs. Mean age of incidence of ALD was 51.38 \pm 5.78 years in patients. It was seen that mean age in both cases and controls was nearly same and no significant difference in age was found. In this study, we found that the difference in the levels of MDA in controls and patients was statistically highly significant with the level of MDA significantly higher in patients as compared to controls. The difference in the levels of GSH in controls and patients were statistically highly significant with the level of GSH significantly decreased in patients as compared to controls. The observations were similar to findings of other investigators like V Dinu [20] and D M Vasudevan [21]. The difference in the levels of AST,ALT,GGT \& AST/ALT ratio in controls and patients were statistically highly significant $(p<0.0001)$ with the levels of all these parameters significantly increased in patients as compared to controls. These results were in accordance with S K DAS [22], Irie et al [23] and Nalpas et al [24]. The prevalence of alcoholic liver disease is influenced by many factors including genetic and environmental factors; however the risk of liver disease increases with the quantity and duration of alcohol intake [25].

A significant negative correlation between MDA and GSH was found ( $p=0.01$ ), which clarifies that high lipid peroxidation depletes antioxidant
GSH. In the present study the cases were divided into 2 groups on the basis of duration of alcohol consumption. We compared levels of various markers discussed above with the duration of alcohol consumption in cases and it was observed that there was no significant variation in various parameters under study with duration of alcohol consumption. It may be due to different amount of alcohol intake, nutritional and comorbidity factors. The observations supported the findings of Shen et all [26] who found no significant dose response relation between daily alcohol intake and abnormal alcohol-related liver injury indicators as well as between duration of drinking and abnormal alcohol-related liver injury indicators. Monitoring GGT, AST and ALT in combination is a sensitive means of detecting severity of alcohol induced liver damage. Increased lipid peroxidation and depletion of reduced glutathione could occur as a consequence of free radical generation due to alcohol consumption. All these parameters in combinations may be useful indicator for identification and determination of severity of alcoholic liver diseases.

\section{CONCLUSION}

The results of the study are suggestive of using serum MDA and Reduced GSH as novel markers for estimating alcohol induced oxidative stress which is compromising the AODS (antioxidant defense system) in the patients of ALD, causing liver injury in the early age of $40-50 \mathrm{yrs}$. It is safe to assume that the most practical means of preventing ALD and delaying its progression from fatty liver to cirrhosis liver, is to educate and increase awareness of large population about the benefits of consuming a diet rich in antioxidants. As apart from abstinence from alcohol, proper nutrition and supplementation of antioxidant are some of the therapeutic options. However, it would be useful to evaluate other aspects of the AODS which were not explored in the present study, such as the antioxidant vitamins, enzymes and also the liver regeneration markers during abstination.

\section{REFERENCES}

[1]. Reuben A. Alcohol and the liver. Curr Opin Gastroenterol 2007;23:283-91. 
[2]. Sussman S, Dent CW, Skara S, de Calice P, Tsukamoto $\mathrm{H}$. Alcoholic liver disease (ALD): a new domain for prevention efforts. Subst Use Misuse 2002;37:18871904.

[3]. National Institute on Alcohol Abuse and Alcoholism. Alcohol Alert No.19: Alcohol and the Liver. PH 329. Rockville, MD: the Institute; 1993.

[4]. Kurose I, Higuchi H, Kato S, Miura S, Ishii H. Ethanolinduced oxidative stress in the liver. Alcoholism: Clinical and Experimental Research 1996;20(1):7785.

[5]. Stewart S, Jones D, Day CP. Alcoholic liver disease: new insights into mechanisms and preventative strategies. Trends Mol Med 2001;7:408-13.

[6]. Zhou Z, Wang L, Song Z, Lambert JC, McClain CJ, Kang YJ. A critical involvement of oxidative stress in acute alcohol-induced hepatic TNF-alpha production. AM J Pathol 2003;163:1137-46.

[7]. Tsukamoto H, Lu Sc. Current concepts in the pathogenesis of alcoholic liver injury. FASEB J 2001;15:1335-49.

[8]. Clarkson PM. Antioxidants and physical performance. Crit Rev Food Sci Nutr 1995;35:131-141.

[9]. Karlsson J. Introduction to Nutraology and Radical Formation. In: Antioxidants and Exercise. Illinois: Human Kinetics Press; 1997. p.1-143.

[10]. Farmer EE, Davoine C. Reactive electrophile species. Curr Opin Plant Biol 2007;10(4):380-6.

[11].Moore K, Roberts LJ. Measurement of lipid peroxidation. Free Radic Res 1998;28(6):659-71.

[12]. Del Rio D, Stewart AJ, Pellegrini N. A review of recent studies on malondialdehyde as toxic molecule and biological marker of oxidative stress. Nutr Metab Cardiovasc Dis 2005;15(4):316-28.

[13].Kaczmarski M, Wojicicki J, Samochowiee L, Dutkiewicz T, Sych Z. The influence of exogenous antioxidants and physical exercise on some parameters associated with production and removal of free radicals. Pharmazie 1999;54:303-06.

[14]. Dekkers JC, van Doornen LJP, Kemper Han CG. The Role of Antioxidant Vitamins and Enzymes in the Prevention of Exercise-Induced Muscle Damage. Sports Med 1996;21:213-238.
[15]. Pressman AH. Glutathione: the Ultimate Antioxidant. New York: St.Martin's Press; 1997.

[16]. Beuge JA, Aust AD. Microsomal Lipid peroxidation. In: methods in enzymology, Academic press, New York; 1978;52:302-310

[17]. Beutler E, West C, Beutler B. Electrophoretic polymorphism of glutathione peroxidase. Annals of Human Genetics 1974;38(2):163-69.

[18]. IFCC methods for the measurement of catalytic concentration of Enzymes. J Clin Chem Clin. Biochem 1986;24:481.

[19]. Portmann B, Theodossi A. The value of liver biopsy in alcoholic liver disease. Alcohol Alcohol 1982;17: 16-31

[20]. Dinu V, Zamfir O. Oxidative stress in ethanol intoxicated rats. Rev Roum Physiol 1991;28(12):63-67.

[21]. Wessner B, Strasser EM, Spittler A, Roth E. Effect of single and combined supply of glutamine, glycine, $\mathrm{N}$-acetylcysteine, and $\mathrm{R}, \mathrm{S}$-alpha-lipoic acid on glutathione content of myelomonocytic cells. Clin Nutr 2003;515-22.

[22]. Das SK, Nayak P, Vasudevan DM. Biochemical markers of alcohol consumption. Ind J Clin Biochem 2003;18(2):111-18.

[23]. Irie M, Suzuki N, Sohda T, Anan A, Iwata K, Takeyama $Y$ et al. Hepatic expression of gamma glutamyltranspeptidase in the human liver of patients with alcoholic liver disease. Hepatol Res 2007;37:966-73.

[24]. Nalpas B, Vassault A, LeGuillou A. Serum activity of mitochondrial aspartate amino transferase: a sensitivity marker of alcoholism with or without alcoholic hepatitis. Hepatology 1984;4:893-96.

[25]. Lelbach WK. Epidemiology of alcoholic liver disease. Prog Liver Dis 1976;5:494-515.

[26]. Shen Z, Li YM, Yu CH, Shen Y, Xu L, Xu CF et al. Risk factors for alcohol related liver injury in the island population of china; A population based case control study. World J Gastroenterol 2008;14(14):225561.

How to cite this article: Pinky Garg, Saroj Choudhary, Montosh Chakraborty, Karthikeyan P. INDICES OF OXIDATIVE STRESS AND LIVER INJURY IN ALCOHOLIC LIVER DISEASE. Int J Intg Med Sci 2017;4(5):488492. DOI: $10.16965 / \mathrm{ijims} .2017 .107$ 